\title{
Visual perception of digital holograms on autostereoscopic displays
}

\author{
Taina M. Lehtimäki, ${ }^{a}$ Kirsti Sääskilahti, ${ }^{a}$ Risto Näsänen, ${ }^{a}$ and Thomas J. Naughton ${ }^{b, a}$ \\ ${ }^{a}$ University of Oulu, RFMedia Laboratory, Oulu Southern Institute, Vierimaantie 5, 84100 \\ Ylivieska, Finland. \\ ${ }^{b}$ Department of Computer Science, National University of Ireland Maynooth, County Kildare, \\ Ireland
}

\begin{abstract}
In digital holography we often capture optically a 3D scene and reconstruct the perspectives numerically. The reconstructions are routinely in the form of a 2D image slice, an extended focus image, or a depth map from a single perspective. These are fundamentally $2 \mathrm{D}$ (or at most 2.5D) representations and for some scenes are not certain to give the human viewer a clear perception of the 3D features encoded in the hologram (occlusions are not overcome, for example). As an intermediate measure towards a full-field optoelectronic display device, we propose to digitally process the holograms to allow them to be displayed on conventional autostereoscopic displays.
\end{abstract}

\section{INTRODUCTION}

In contrast to conventional holography, ${ }^{1}$ in digital holography ${ }^{2-6}$ we often capture optically a 3D scene and reconstruct the desired perspectives numerically in a computer. Numerical reconstruction provides a significant amount of flexibility since the hologram data is in a convenient form for such digital image processing, there is a wealth of image processing technology available, and these technologies can be readily extended. The reconstructions are routinely in the form of a $2 \mathrm{D}$ image slice through the reconstruction volume, an extended focus image, or a depth map from a single perspective. These are fundamentally $2 \mathrm{D}$ (or at most 2.5D) representations and for some scenes are not certain to give the human viewer a clear perception of the 3D features encoded in the hologram (occlusions are not overcome, for example). Conveniently and automatically extracting 3D information from digital holograms remains problematic in the general case. Alternatively, an optical reconstruction would allow the human viewer to extract the 3D information themselves through stereopsis and motion parallax, but convincing full-field digital holographic displays for real-world 3D scenes have not been demonstrated due to current limitations of programmable display devices. ${ }^{7,8}$ As an intermediate measure, we propose to digitally process the holograms to allow them to be displayed on conventional autostereoscopic displays. The digital capture and digital image processing provides the flexibility required, the conventional autostereoscopic displays provide a readily understood and off-the-shelf available technology, and the human visual system itself extracts the 3D information of the scene encoded in the hologram. We explain how this approach allows for a systematic study of human visual perception of some properties of digital holograms of real-world objects and present extensions to our first results. ${ }^{9}$

\subsection{Perceptual studies with digital holograms}

The depth perception of images produced by digital holography is affected by image quality (e.g. the presence of noise, blur, and compression artefacts) and the method of how images are displayed to the viewer (e.g. ordinary display or stereoscopic display with or without the possibility to dynamically change perspective). The challenges in visualising digital holograms of real-world 3D objects are (1) large calculation times required for the reconstruction of different perspectives and different depths for large holograms (for example calculating an extended focus image ${ }^{10,11}$ ), (2) the amount of noise (speckle and otherwise) present in the reconstructions, ${ }^{12}$ and

Email: firstname.lastname@oulu.fi

Three-Dimensional Imaging, Visualization, and Display 2009,

edited by Bahram Javidi, Jung-Young Son, Manuel Martinez-Corral, Fumio Okano, Wolfgang Osten,

Proc. of SPIE Vol. 7329, 73290C · @ 2009 SPIE · CCC code: 0277-786X/09/\$18 · doi: 10.1117/12.821864

Proc. of SPIE Vol. 7329 73290C-1 
in the context of stereoscopic display (3) the fact that only one depth plane is in focus in each reconstruction given that good stereo perception requires high spatial frequencies (a small amount of blurring) in the images. ${ }^{13,14}$

Depth perception is based on monocular and binocular cues as well as self motion (motion parallax). Monocular cues are texture gradients, shading, geometrical perspective, and occlusions. Binocular, i.e. stereoscopic, depth cues are produced by differences, i.e. disparities, between the images formed on the retinae of the two eyes. The displaying method as well as image quality can affect the visibility of these cues. Our aim is to develop and apply robust experimental methods for the evaluation of perceptual image quality as well as for the evaluation of the effectiveness of various displaying methods in the context of digital holography. One approach is to measure human performance (e.g. speed and accuracy) in perceiving depth information. Perceptual performance can reflect the amount of information that can be transmitted and interpreted by the human visual system. For example, stereoscopic displaying of images or the use of motion may ease depth perception and improve its accuracy or increase the speed by which 3D shapes can be recognised.

Perceptual performance can be effectively measured by psychophysical methods such as the threshold estimation techniques e.g. the staircase algorithm. By threshold estimation methods one can measure e.g. the minimum amount of disparity that can be discerned with a given probability or the minimum time required for the recognition of a 3D object. Psychophysical threshold estimation methods produce highly accurate and quantitative ratio scale level descriptions of human performance.

Subjective evaluations, such as scaling of quality and subjective naturalness of perception, mean opinion scores, questionnaires, and interviews can be used to gather complementary information about observers' experiences, which cannot be evaluated by psychophysical methods.

The purpose of our particular study is to find out if is it possible to overcome the challenges of visualising digital holograms in a way that allows people to use their natural stereoscopic 3D perception. In first part of our study we use digital holograms of objects with short depth which allows us to avoid the blurring effect in some parts of the reconstructions of the digital holograms. In the second part of the study, we examine the effects of changing the window size for stereoscopic perception of digital holograms. In the third part of our study we look further into the possibilities of combining a stereoscopic display with digital holograms to assess the limits of human perception with holograms encoding objects of greater depth.

\subsection{Human stereovision}

Stereovision is one principal way humans extract 3D information from a scene. Both eyes get slightly different images due to their different positions in the head. The human vision system is able to combine these different images forming a 3D perception of the scene: a process called stereopsis. It has been shown that typically the higher spatial frequencies are the most important for perception of $3 \mathrm{D}$ objects (meaning that stereo acuity declines when the amount of blurring increases).$^{14}$ However, a study conducted by Papelba et al. ${ }^{13}$ with disbalanced images suggests that within certain limits it is possible to perceive stimuli in $3 \mathrm{D}$ even when one eye is seeing a blurred image and one eye a focussed image.

In our study we also use disbalanced images, but due to the nature of the reconstructions of digital holograms, our stimuli are focussed for each eye at a different depth, since blurring in a digital hologram reconstruction corresponds to an incorrect depth reconstruction. For each stereo pair, we reconstruct two scenes from the digital hologram at different depths (for example one focussed at the front-most part of the scene and the other focused at the back-most part) and determine the quality of the stereo perception.

\subsection{Capturing the holograms}

Our digital holograms are recorded using an optical setup based on a Mach-Zehnder interferometer architecture in an in-line configuration. A spatially filtered linearly polarized helium neon $(632.8 \mathrm{~nm})$ laser beam is split into object and reference beams, both of which are spatially filtered and expanded. The first beam illuminates the 3D object placed at a distance $d$ from a 10-bit $2032 \times 2048$ pixel CCD camera. The reference beam passes through half-wave plate RP1 and quarter-wave plate RP2. Through permutation of the fast and slow axes of the plates we can achieve phase shifts of $0,-\pi / 2,-\pi$, and $-3 \pi / 2$. The reference beam combines with the light diffracted 


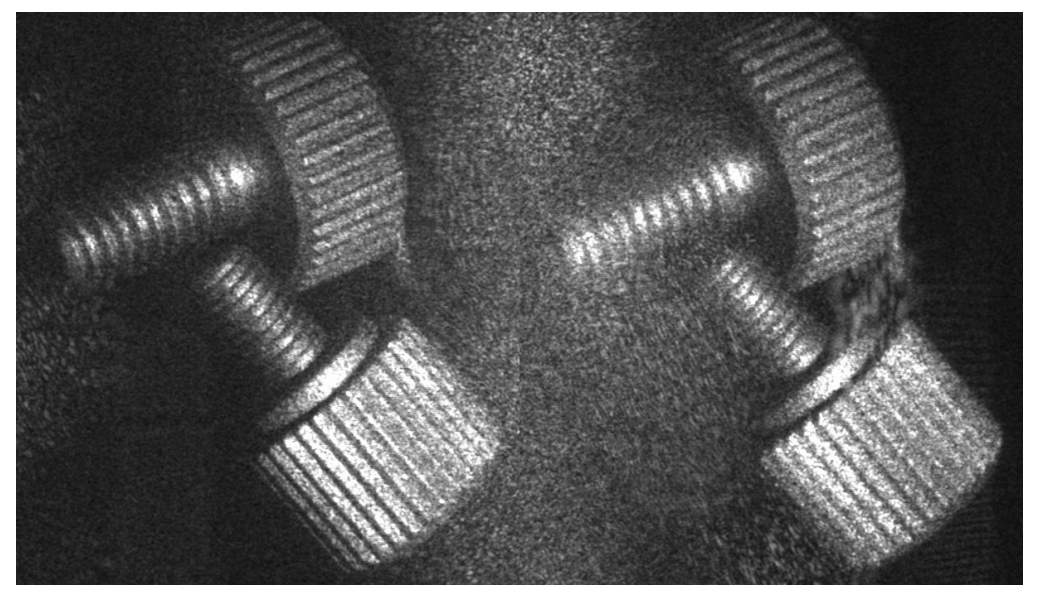

Figure 1. Left and right perspectives of a scene where almost all parts of the scene are in focus.

from the object and forms an interference pattern in the plane of the camera. At each of the four phase shifts we record an interferogram. Using these four intensity images, the complex-valued camera-plane wavefront can be approximated in a computer to good accuracy using phase-shift interferometry. ${ }^{15,16}$ Digital holograms of reasonably diffuse $3 \mathrm{D}$ objects were used. The objects had approximate dimensions of $5 \mathrm{~mm} \times 5 \mathrm{~mm} \times 5 \mathrm{~mm}$ and were positioned approximately $d=350 \mathrm{~mm}$ from the camera.

A perspective of the object encoded in the digital hologram is reconstructed by numerically simulating the propagation of light backwards in time and space. By propagating a distance $-d$ one reconstructs a perspective with all points of the object at distance $d$ in focus, and all other points out of focus proportional to their distance from $d$. By propagating the full hologram field one creates an on-axis perspective. By propagating a subset of pixels, and employing the appropriate linear phase tilt, one creates a (lower-resolution) perspective determined by the distance from the centre of this subset to the optical axis.

The range of viewing angles is determined by the ratio of the window size to the full CCD sensor dimensions. Our CCD sensor has approximate dimensions of $18.5 \mathrm{~mm} \times 18.5 \mathrm{~mm}$ and so a $1024 \times 1024$ pixel window has a maximum lateral shift of $9 \mathrm{~mm}$ across the face of the sensor. With an object positioned $d=350 \mathrm{~mm}$ from the camera, viewing angles in the range $\pm 0.74^{\circ}$ are permitted. Smaller windows will permit a larger range of viewing angles at the expense of image quality at each viewpoint.

\section{METHODS}

\subsection{Subjects and apparatus}

Experiments one and three were performed using six subjects. Their stereovision was tested with stereo pair photographs before the experiment to ensure they had good stereovision acuity. The second experiment was performed with thirteen subjects with normal or corrected-to-normal vision. Stimuli were presented on a Sharp LL-151-3D LCD monitor. This is an autostereoscopic display; it allows users to see stereoscopic images without using special glasses. It consists of two LCD panels, one in front of another. In 3D mode the foreground LCD panel acts as a parallax barrier so that the right and left eyes of a viewer receive different images. The display resolution was $1024 \times 768$ pixels $(512 \times 768$ pixels for each eye) and the distance between the subject and display was approximately $60 \mathrm{~cm}$.

\subsection{Stimuli}

We used two types of stimuli to investigate if our subjects were able to see the stimuli in 3D. First we experimented with our idea of perception in 3D despite the noise in the reconstructions. For Experiment 1 we used digital holograms of objects with very small depth. With these holograms it was possible to create reconstructions at 
(a)

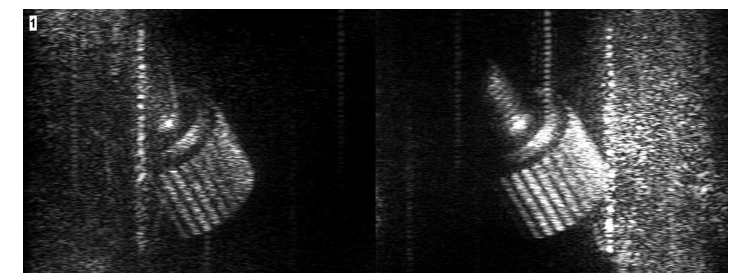

(b)

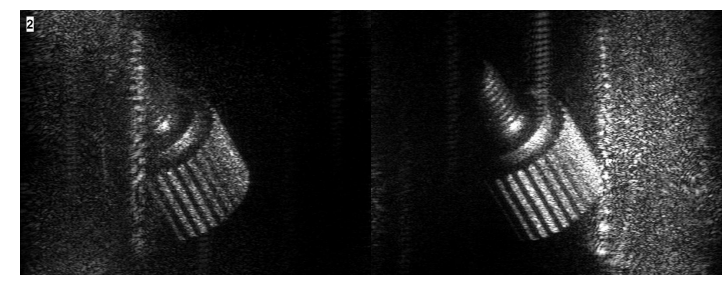

(c)

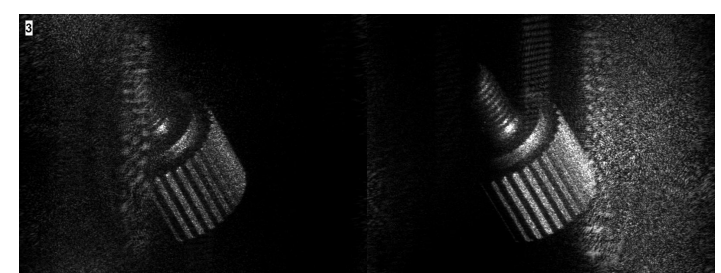

(d)

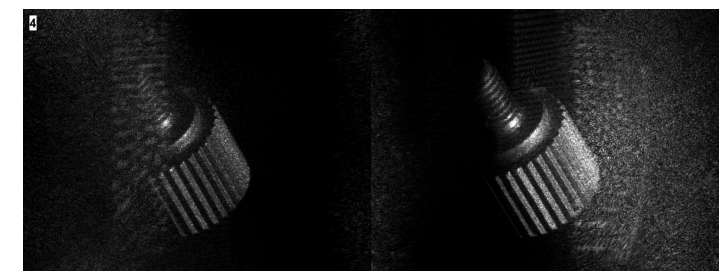

(e)

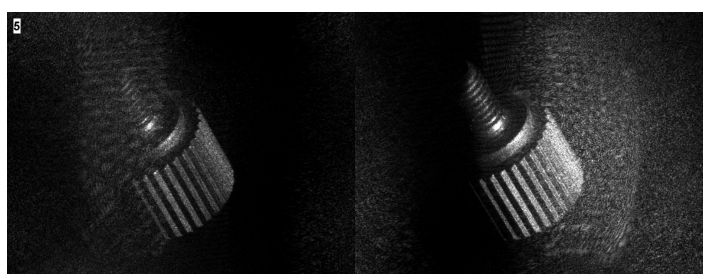

(f)

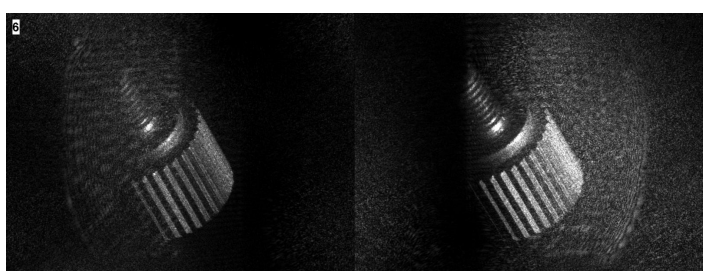

Figure 2. Reconstructed stereo pairs using different window sizes from a particular digital hologram: (a) $64 \times 64$ pixels, (b) $128 \times 128$ pixels, (c) $256 \times 256$ pixels, (d) $512 \times 512$ pixels, (e) $768 \times 768$ pixels, and (f) $1024 \times 1024$ pixels.

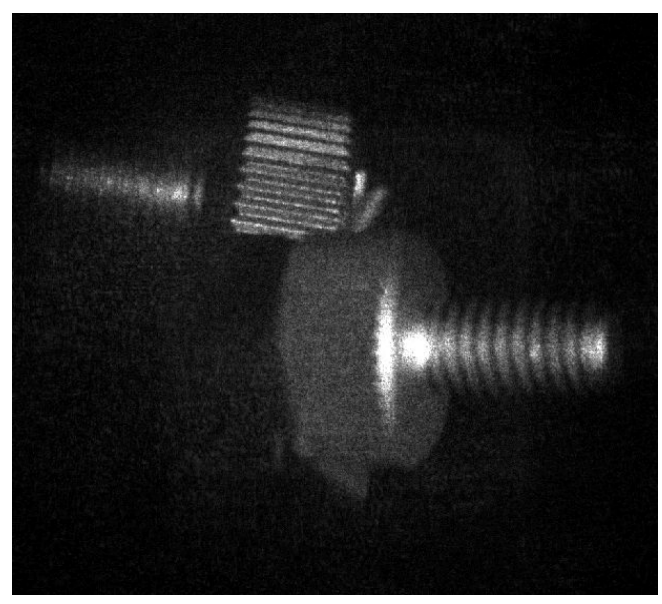

(a)

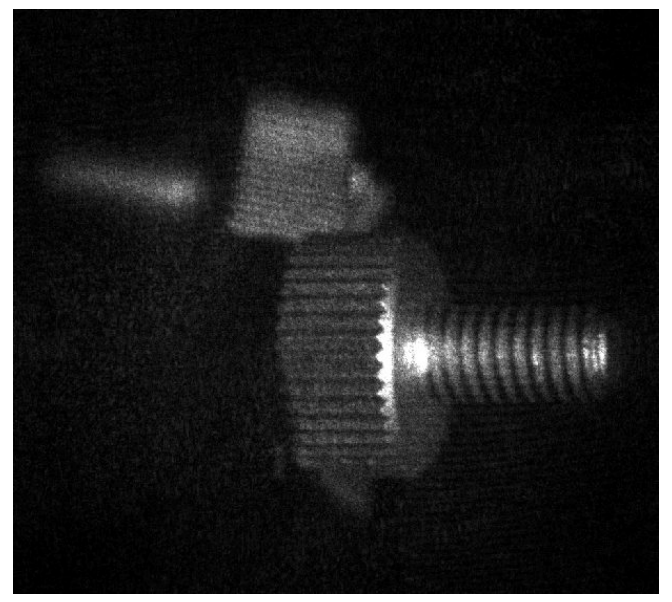

(b)

Figure 3. Disbalanced stereo pair: top, back of scene in focus; bottom, front of scene in focus.

only one depth that still had the whole image appear largely in-focus to the viewer. In Fig. 1 we show an example of our stereo pair stimuli of an object with small depth. Our stereo pairs consisted of reconstructions made with window sizes from 64 pixels to 1024 pixels. Stereo pairs were created by taking reconstructions using left and right windows in the hologram plane corresponding to left and right perspectives of the object. Mean filtering was applied to reduce noise.

For Experiment 2, we presented subjects with reconstructions from five different holograms, using six different sized windows from each hologram. Figure 2 shows the result of reconstructing one of the holograms with different window sizes. Again, mean filtering was applied to each. 

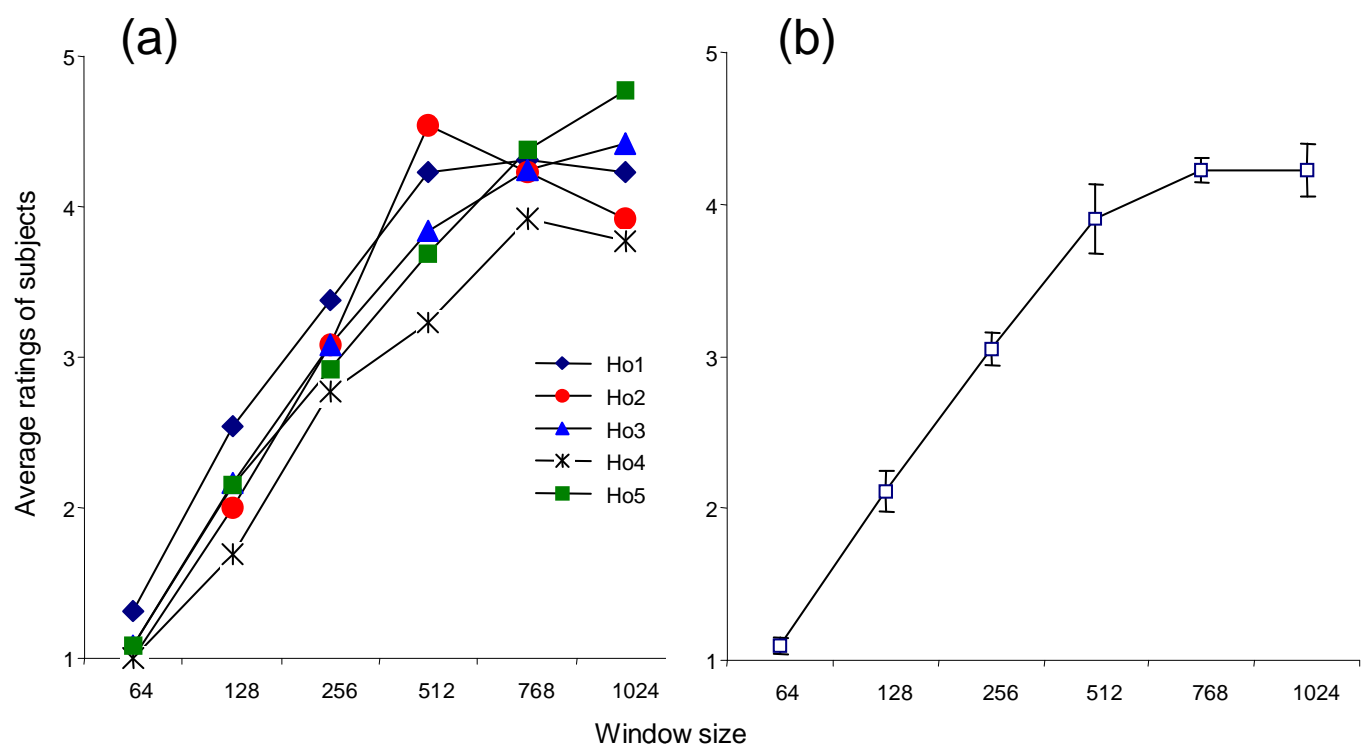

Figure 4. The percieved quality of holograms using scale 1-5: (a) for individual holograms, and (b) averages over all holograms. The quality increases linearly from window size $64 \times 64$ pixels to size $512 \times 512$ pixels, after which size the quality remains constant.

For Experiment 3 we used disbalanced stimuli reconstructed from a hologram of the two-object scene shown in Fig. 3. We chose $512 \times 512$ pixel window size: a choice informed from the results of Experiment 1 . In these stimuli, part of the image was blurred according to the reconstruction depth, as shown in Fig. 3. We used in total ten reconstructions (five different depths labelled $\mathrm{f}, \mathrm{mf}, \mathrm{m}, \mathrm{mb}$, and $\mathrm{b}$ ranging from the front in focus $\mathrm{f}$ to the back in focus $b$, for each of left and right perspectives). From these we assembled 9 stereo pairs, the labels of which are listed in Fig. 5, where fb means in the left perspective the front of the scene was in focus and in the right perspective the back was in focus.

\subsection{Procedure}

In Experiment 1 we first asked subjects to grade the subjective quality of individual reconstructions presented on a conventional 2D display. Subjects were instructed to form their own opinion of high/low quality but to remain consistent throughout the experiments. They were asked to use a scale from 1 (very poor) to 9 (excellent). Next we asked them to grade stereo pairs presented on the autostereoscopic display according to the subjective quality of the stereo image and separately the amount of 3D effect perceived. For Experiment 2, we asked subjects to evaluate the quality of each image using a scale frin 1 (very poor) to 5 (very good). For Experiment 3 we first asked subjects to grade the amount of focus in the ten individual reconstructions for the front and back object separately. We then asked them to do the same for the nine stereo pairs presented on the autostereoscopic display.

\section{RESULTS}

Despite the noise in the reconstructions and their general poor quality, consistent with all digital holograms using current technology, the stereo pairs were perceived in 3D by all test subjects. The quality of 3D perception and image quality depended on the reconstruction window size but only up to $512 \times 512$ pixel windows; likely due to our speckle reduction filtering. When a stereo pair is presented to a subject, and where individually that subject has classed each of the two images as having different subjective quality, our subjects consistently rated the quality of the stereo pair as being as good as the better of the two images.

The results from Experiment 2 are shown in Fig. 4 as average ratings over the thirteen subjects, and for five different holograms. The quality increases linearly from side length 64 to side length 512, after which size the 

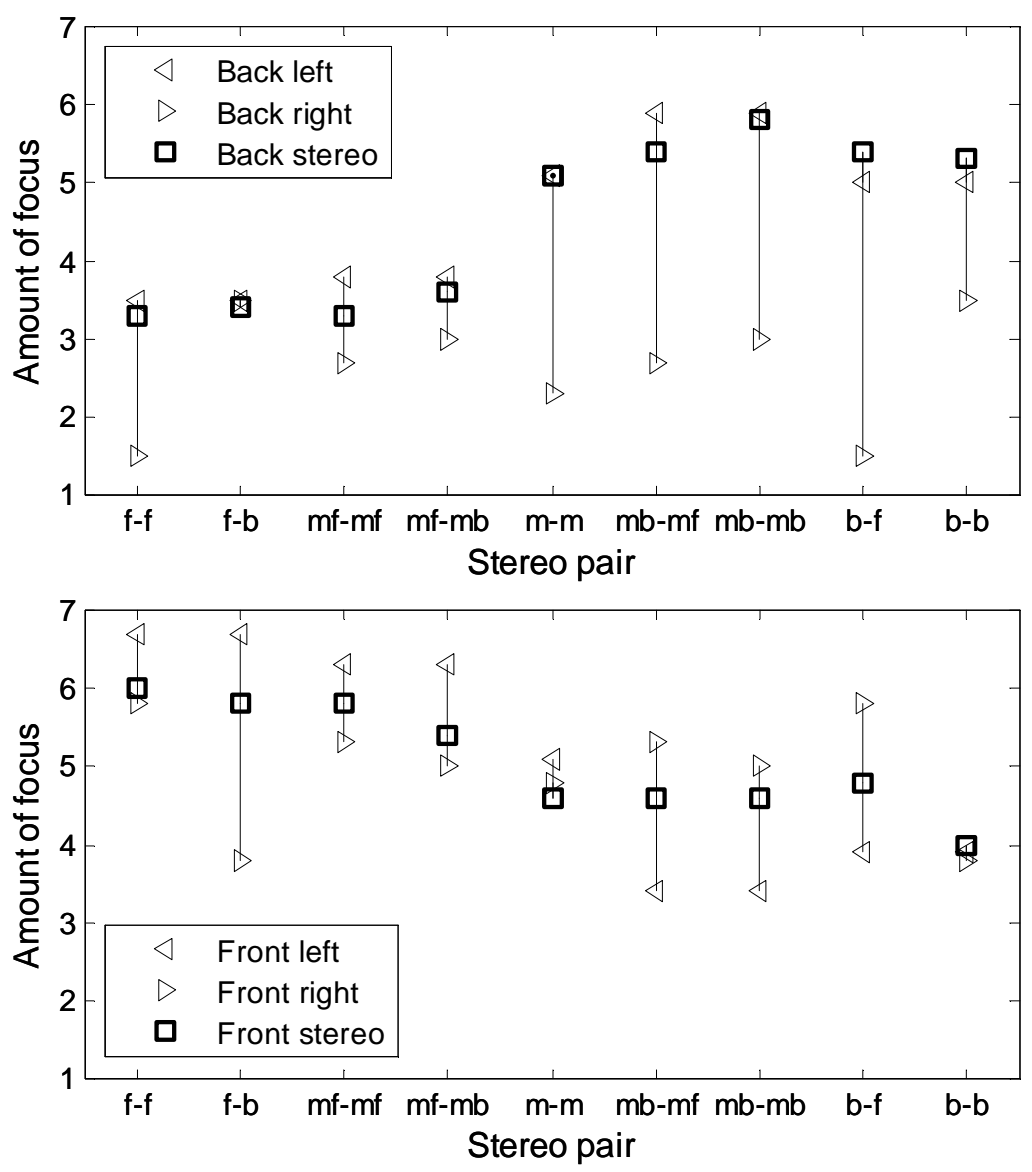

Figure 5. Amount of perceived focus in the back object (upper) and front object (lower) in the left and right individual reconstructions, and in the stereoscopically viewed pair, for each of nine stereo pairs. Average grades of all subjects plotted. Perceived focus in stereo is at least as good as the average of the in and out of focus versions.

quality remains at the same level, again, likely due to the effect of our mean filtering.

In Experiment 3, the stereo pairs were still perceived in 3D when one eye was presented with a reconstruction from the front of the scene and the other eye presented with a reconstruction from the back of the scene. However 2 out of 6 subjects consistently graded the 3D effect as being poor. The subjects' perceived amount of focus in individual reconstructions and in stereoscopically perceived image are shown in Fig. 5. When shown a disbalanced pair in stereo, subjects consistently perceived each object to be more in focus than its out-of-focus equivalent in individual reconstructions. Compared to an individual reconstruction where the back or front object was out of focus, the disbalanced stereo pair showed a mean increase in the back object of 1.8 and in the front object of 0.7. In both cases, the average was beaten.

\section{DISCUSSION}

There are implications for this work in terms of digital holographic video. If one uses a 2D monitor to display the intensity of a reconstruction from a digital hologram one needs to display a sequence of such $2 \mathrm{D}$ reconstructions to allow different perspectives to be viewed. To an observer this sequential visualization is perceived as rotation of the object. However, if movement is used to convey 3D information about the object then this approach is limited to viewing static 3D scenes. By using stereoscopic visualization we can retain this time dimension in digital holographic display. We can take a sequence of holograms of a moving object and in this way display digital holographic videos. Alternatively, the time dimension could be retained for some other purpose such 
as story telling. Furthermore, in terms of also presenting different perspectives to each eye, we find that our approach more efficiently allows for 3D perception of objects encoded in digital holograms by not requiring an extended-focus perspective to be generated for each eye.

\section{ACKNOWLEDGEMENTS}

We thank Conor Mc Elhinney for capturing the digital holograms. We acknowledge support from Science Foundation Ireland under the National Development Plan, a European Commission Marie Curie Intra-European Individual Fellowship, and the European Community's Seventh Framework Programme FP7/2007-2013 under grant agreement no. 216105 .

\section{REFERENCES}

1. D. Gabor,"A new microscopic principle," Nature, vol. 161, pp. 777-778, 1948.

2. J.W. Goodman and R.W. Lawrence, "Digital image formation from electronically detected holograms," Applied Physics Letters, vol. 11, pp. 77-79, 1967.

3. M.A. Kronod, N.S. Merzlyakov, and L.P. Yaroslavskii, "Reconstruction of a hologram with a computer," Sov. Phys. Tech. Phys, vol. 17, p. 333, 1972.

4. U. Schnars and W. Jueptner, Digital Holography: Digital Hologram Recording, Numerical Reconstruction, and Related Techniques, Springer, 2005.

5. Th. Kreis, Handbook of Holographic Interferometry: Optical and Digital Methods, Wiley, 2005.

6. L. Onural and P.D. Scott, "Digital decoding of in-line holograms," Optical Engineering, vol. 26, pp. 1124$1132,1987$.

7. M. Sutkowski and M. Kujawinska, "Application of liquid crystal (LC) devices for optoelectronic reconstruction of digitally stored holograms," Optics and Lasers in Engineering, vol. 33, pp. 191-201, 2000.

8. O. Matoba, T.J. Naughton, Y. Frauel, N. Bertaux, and B. Javidi, "Real-time three-dimensional object reconstruction by use of a phase-encoded digital hologram," Applied Optics, vol. 41, pp. 6187-6192, 2002.

9. T.M. Lehtimäki and T.J. Naughton, "Stereoscopic viewing of digital holograms of real-world objects," 3DTV-Conference 2007 - Capture, Transmission and Display of 3D Video, pp. 1-4, Kos, Greece, 7-9 May 2007.

10. P. Ferraro, S. Grilli, D. Alfieri, S. De Nicola, A. Finizio, G. Pierattini, B. Javidi, G. Coppola, and V. Striano, "Extended focused image in microscopy by digital holography," Optics Express, vol. 13, 6738-6749, 2005.

11. C.P. McElhinney, B.M. Hennelly, and T.J. Naughton, "Extended focused imaging for digital holograms of macroscopic three-dimensional objects," Applied Optics, vol. 47, pp. D71-D79, 2008.

12. J.C. Dainty, "Laser Speckle and Related Phenomena", Springer Verlag, Berlin, second edition, 1984.

13. G. Papelba, I. Cipane, and M. Ozolinsh, "Stereovision studies by disbalanced images," Advanced Optical Devices, Technologies, and Medical Applications, Proceedings of SPIE vol. 5123, pp. 323-329, 2003.

14. L.M. Wilcox, J.H. Elder, and R.F. Hess, "The effects of blur and size on monocular and stereoscopic localization," Vision Research, vol. 40, pp. 3575-3584, 2000.

15. I. Yamaguchi and T. Zhang, "Phase-shifting digital holography," Optics Letters, vol. 22, pp. 1268-1270, 1997.

16. Y. Frauel, E. Tajahuerce, M.A. Castro, and B. Javidi, "Distortion-tolerant 3D object recognition using digital holography," Applied Optics, vol. 40, pp. 3887-3893, 2001. 